\title{
"The health of the nation": a challenge for genitourinary medicine
}

The Health of the Nation ${ }^{1}$ was published in June, 1991. It was designed as a consultation document, presenting the Government's proposals towards developing a health strategy for England; similar documents are planned for Scotland, Wales and Northern Ireland.

Genitourinary medicine has an important public health role and the document must and should have important implications for the specialty. Unfortunately, this may not prove to be so, since AIDS and sexually transmitted diseases do not feature as priorities.

\section{The Health of the Nation}

The main statements of the document concern:

\section{(1) Key strategic role of Health Authorities}

Each Health Authority will aim to achieve a coordinated health strategy for its local population, in response to local need and in line with nationally agreed directives.

\section{(2) Balancing prevention and clinical care}

The document emphasises health promotion; it urges that policymaking be stated more strongly in terms of prevention of illness and avoidable premature death.

\section{(3) Collaboration}

Responsibility for health is wide; achieving better health requires the collaboration of many individuals and agencies.

\section{(4) The challenges}

Both preventable ill health and the significant variations in health (geographical, ethnic, social and occupational) which exist within the nation are the major challenges for the future.

\section{(5) The Key Areas}

The document proposed that key areas be prioritised for health improvement. Three criteria determine selection of a key area. Firstly, the area should be a major cause of premature death or avoidable ill-health, either in the population as a whole or amongst specific groups of people. Secondly, effective interventions should be possible. Thirdly, it should be possible to set objectives and progress towards them should be monitorable by specific indicators.

The paper suggested 16 candidates as possible key areas, including HIV/AIDS, but then went on to exclude four of them (one of which was also HIV/AIDS) because they did not fulfil all the necessary criteria for inclusion.

\section{Implications for HIVIAIDS services}

HIV/AIDS narrowly missed being defined as a "key area". It was initially selected as a strategic priority, only to be later rejected. The explanation given for this was that Criterion 3 for determining a "key area", the ability to set specific targets and to monitor progress towards them, was not met. The document did not expand much on this, stating only that "more needs to be known about the current prevalence of the disease. The current series of anonymised serosurveys may help to provide further information".

From a document which claims to champion prevention, this view is surprisingly narrow and lacking in foresight. There is no vaccine or cure for HIV disease.
Prevention is the only control strategy we have and to be effective it needs to be early. Delaying action for the sake of obtaining more detailed data on disease incidence is unlikely to be productive.

The whole question of target-setting in the context of HIV/AIDS can be reconsidered, expanding the horizons beyond seroprevalence statistics. A broader approach to this subject was taken by a recent working party set up by the Faculty of Public Health Medicine of the Royal Colleges of Physicians. The working party was established to validate indicators of levels of health, investigate the current state of the nation's health and suggest targets for improvement. It published its first report in June, 1991. ${ }^{2}$

In discussing target-setting, it states that targets can usefully highlight important areas of strategy, convert theoretical policy into practical programmes and provide tangible indicators of progress. They do, however, have pitfalls; they may lead to spurious priority being given to measurable objectives and they may grossly oversimplify health policy. Aware of their limitations, the report went on to suggest that targets be considered from four perspectives:

(i) improved health status

(ii) risk factor reduction

(iii) improved services and protection

(iv) surveillance and data needs.

This broader perspective could usefully be applied to target setting for HIV/AIDS.

\section{(i) Improved health status}

HIV control programmes aim to reduce future virus spread and prevent new cases of infection. Their ultimate goal is improved health status of the population and this is the best outcome measure of their success. Setting numerical targets for health status does, however, require baseline information such as seroprevalence data.

The national anonymised HIV seroprevalence study currently being coordinated by the Communicable Diseases Surveillance Centre has already reported interim results which indicate continuing high seroprevalence rates in homosexual men attending genitourinary medicine (GUM) clinics in London; ${ }^{3}$ among antenatal patients in the Thames Regions, seroprevalence rose fourfold between 1988 and 1990 , to a level of $1: 500 .^{4}$ In the near future, further information from this study could certainly form a basis for numerical seroprevalence targets in defined population groups. The Government's dismissal of this accumulating knowledge would seem unduly pessimistic.

\section{(ii) Risk factor reduction}

Measurement of risk factors for HIV transmission presents a number of opportunities for setting targets. Indicators of safer sexual behaviour and drug injecting practices are readily identifiable; for some population subgroups, much clinical and epidemiological data is already being informally collected, by GUM clinics and drug projects.

The KC60 system could be readily modified to include sexual behaviour indices, such as age at coitarche, number of partners in the previous 12 months, duration of relationships, condom use with non-regular partners, type of sexual activities practised and sexual contacts abroad. These data could form the basis of targets for risk factor reduction.

Similarly, amongst injecting drug users attending drug 
treatment agencies, information could readily be obtained on age at initiation of drug use, needle-sharing activity (including geographical location) and could also include basic sexual behaviour data, such as condom use at last sexual intercourse.

The problem about these data, however, is that they are not representative of the population at large, on whom there are practically no baseline data on sexual and drugusing behaviour. However, there are moves towards rectifying this problem; both the National Survey of Sexual Attitudes and Lifestyles ${ }^{5}$ and a number of surveys on sexual knowledge, attitudes and behaviour undertaken by the Health Education Authority ${ }^{6}$ will in the near future provide data for baseline estimates and target setting. The considerable geographical variation in risk factors for HIV infection means that local adjustments to national guidelines for targets will be required.

Risk factor reduction is achieved by education. Performance indicators can be applied to educational programmes and targets for future improvement set. HIV/AIDS education has a broad framework, including media campaigns, schools and higher education institutions, health care providers and the police and prison services. National monitoring of the extent and success of formal HIV education policies as applied in these various situations would be a feasible means of target setting.

(iii) Improved services

The role of GUM clinics is crucial in both prevention and treatment of HIV disease. Fundamental to the success of a District Health Authority's HIV prevention strategy is an accessible GUM service which takes an active public health role, offering HIV education, counselling and testing to the local population. Developments in earlier intervention with prophylactic therapies in HIV infected patients makes the medical advantages of testing more clearcut than previously. This has led to calls for more widespread testing of the population, which would be most appropriately based in GUM clinics.

Performance indicators for these activities could, at their most basic, consist of numerical workload returns for patients counselled and tested. There are greater opportunities here, however. GUM services are uniquely placed to play a leading role in developing a local HIV prevention policy, providing services in coordination with other related specialists, including obstetricians and gynaecologists, family planning clinics, drug agencies and general practitioners. The extent to which Health Authorities have implemented a locally relevant written policy on HIV prevention could be measured and targets developed.

In terms of clinical care for patients who are already HIV infected, local strategies need to be clearly defined, coordinated and critically evaluated. Standards in both process and outcome of clinical care should be defined; targets for the future can be incorporated. The district audit systems already in existence are ideally placed for use in target setting.

\section{(iv) Surveillance and data needs}

Many of the data systems necessary to monitor and evaluate HIV/AIDS prevention and treatment strategies are already in place. The KC60 system could be refined and improved by incorporating data on sexual behaviour and HIV prevention workload. The national anonymised HIV serosurveys, ${ }^{3}$ the National Study of Sexual Attitudes and Lifestyles ${ }^{5}$ and the regional and national drug databases are all established and will provide much useful data as the basis for targets. Local audit systems already provide an appropriate forum for analysis of clinical activity. Data collection facilities thus appear adequate, with some modifications and the application of sound management skills.

\section{GUM services other than HIVIAIDS}

The Health of the Nation did not make specific reference to sexually transmissible diseases (STDs) apart from HIV/ AIDS, but STD control is closely linked both clinically and epidemiologically to HIV prevention.

For many years, GUM was regarded as a low health care priority and suffered from considerable underresourcing. The arrival of HIV/AIDS led to resources and subsequent improvements in services. There is a danger that the accelerating clinical HIV workload now being felt by GUM services in some areas will not be met by a parallel increase in funding. Patients with other STDs will then once again be faced with an inadequate and unacceptable service which they will not use. The sequelae of STDs include pelvic inflammatory disease, congenital infection and neoplasia; failure to control STDs would have disastrous implications for public health. Genitourinary physicians must ensure that their commitment to HIV/AIDS continues, but not at the expense of the wider range of services which the specialty now offers to its patients.

If HIV/AIDS were to be designated a "key area" in the terms of The Health of the Nation, it would be logical and necessary to include GUM as an integral part of the strategy. This is a good opportunity to reassess other GUM services. Targets for improved health status could be readily identified and indeed have already been published by the Faculty of Public Health Medicine. ${ }^{2}$ Risk factor reduction could be assessed in tandem with sexual behaviour indicators for HIV risk. Improved services for prevention and treatment of STDs were specifically addressed in the Monks Report, ${ }^{7}$ which made over 30 recommendations for improving GUM services in England. Implementation of these recommendations would be an ideal target for future service provision. Lastly, surveillance and data collection in GUM, although predating that in other specialties and countries, could be improved. KC60 clinic returns would benefit from being refined, more standardised and more rapidly processed to allow their more effective use in both epidemiology and in evaluation of services.

The overall spirit of The Health of the Nation presents exciting challenges for genitourinary medicine. It offers opportunities to participate in a coordinated, collaborative health strategy with clear objectives and tangible measurements of success. Preventive medicine is accorded an important role and the major causes of preventable illhealth are approached in a comprehensive manner for the first time. It would be a tragedy if HIV/AIDS and genitourinary medicine were not accorded the priority they deserve.

A SCOULAR

Department of Genitourinary Medicine, $M$ W ADLER

The Middlesex Hospital, London W1N 8AA, UK

1 Secretary of State for Health. The Health of the Nation. London: HMSO, 1991

2 The Faculty of Public Health Medicine of the Royal Colleges of Physicians. UK levels of health: first report, June 1991. London: FPHM, 1991.

3 Public Health Laboratory Service AIDS Centre and Viral Research Laboratory, Academic Department of Genitourinary Medicine, University College and Middlesex School of Medicine and collaborators. The versity College and Middlesex School of Medicine and collaborators. The unlinked anonymous HIV prevalence monitoring programme in England and Wales: preliminary results. Communicable Disease Report 1991; 1:R69-R7

4 Ades AE, Parker S, Berry T, et al. Prevalence of maternal HIV-1 infection in Thames regions: results from anonymous unlinked neonatal testing. Lancet 1991;337:1562-5.

5 Wellings K, Wadsworth J, Field J, Anderson RM, Bradshaw SA. Sexual lifestyles under scrutiny. Nature 1990;348:276-8.

6 Health Education Authority. AIDS strategic monitor: report on the survey period November 1987-December 1988. London: HEA, 1988

7 Working Group to examine Workloads in Genitourinary Medicine Clinics. Report. London: Department of Health, 1988.

Accepted for publicaton 19 March 1992. 